\title{
Adaptive Risk Refinement Methodology for Gas Turbine Engine Rotor Disks
}

\author{
Jonathan P. Moody ${ }^{1}$ and Harry R. Millwater ${ }^{2}$ \\ University of Texas at San Antonio, San Antonio, TX, 78249 \\ Michael P. Enright ${ }^{3}$ \\ Southwest Research Institute, San Antonio, TX, 78238
}

\begin{abstract}
Probabilistic fracture mechanics is a well-established method for predicting the probability-of-fracture (POF) of gas turbine engine rotor disks subject to low-cycle fatigue. An adaptive risk refinement methodology (ARRM) was developed to automate zone discretization and refinement that are typically associated with this approach. ARRM generates initial meshes using an adaptive nodal selection feature designed to optimize accuracy and efficiency. Adaptive mesh refinement (AMR) is performed until a converged risk solution is obtained. ARRM employs several techniques including skeletonization, constrained Delaunay triangulation (CDT), superparametric interpolation, and adaptive mesh refinement. A numerical example is provided to illustrate the effectiveness of the methodology.
\end{abstract}

\section{Nomenclature}

$\begin{array}{ll}\Delta \sigma & =\text { stress range } \\ d_{s} & =\text { shifting distance } \\ F & =\text { fracture of an initial anomaly located in a zone } \\ K & =\text { number of zones } \\ l_{e} & =\text { projection path length } \\ m & =\text { iteration number } \\ P\left[A_{i}\right] & =\text { probability of having an anomaly in a zone } \\ P\left[B_{i} \mid A_{i}\right] & =\text { conditional probability-of-fracture given an anomaly in a zone } \\ P\left[F_{i}\right] & =\text { probability-of-fracture of an initial anomaly located in a zone } \\ P_{\text {disk }} & =\text { probability-of-fracture in a disk } \\ \text { POF } & =\text { probability-of-fracture }\end{array}$

\section{Introduction}

Drobabilistic fracture mechanics is a well-established approach to assess the probability-of-fracture (POF) of turbine engine rotor disks subject to low-cycle fatigue. ${ }^{1,2}$ This methodology utilizes a zone-based risk integration scheme to account for the uncertainty of location of randomly distributed anomalies within a disk. A zone is a grouping of material that has a generally uniform stress state, similar fatigue crack growth properties, inspection schedules, and distribution of anomalies. The risk contribution from each zone is based upon the life limiting location to ensure a conservative risk solution.

The POF of a zone $P\left[F_{i}\right]$ is given by

$$
P\left[F_{i}\right]=P\left[A_{i}\right] \cdot P\left[B_{i} \mid A_{i}\right]
$$

where $P\left[A_{i}\right]$ is the probability of an anomaly in zone $i$, and $P\left[B_{i} \mid A_{i}\right]$ is the conditional POF given an anomaly in zone $i$. The POF of the entire disk $P_{\text {disk }}$ is the probability union of the zone risks:

\footnotetext{
${ }^{1}$ Research Assistant, Dept. of Mechanical Engineering, One UTSA Circle, San Antonio, TX 78249.

${ }^{2}$ Associate Professor, Dept. of Mechanical Engineering, One UTSA Circle, San Antonio, TX 78249.

${ }^{3}$ Principal Engineer, Reliability and Materials Integrity, 6220 Culebra Rd., San Antonio, TX 78238, Member AIAA.
} 


$$
P_{\text {disk }}=P\left[F_{1} \cup F_{2} \cup \cdots \cup F_{K}\right]=1-\prod_{i=1}^{K}\left(1-P\left[F_{i}\right]\right)
$$

where $K$ is the number of zones and $F_{i}$ indicates fracture of an initial anomaly located in zone $i$.

The accuracy of the disk risk value is dependent upon the number of zones. A coarse discretization of zones yields a less accurate result compared to a fine one. The zone mesh is refined through an iterative process. Figure 1 shows a sequence of the zone refinement process for an example rotor disk in which the shaded regions indicate zones that require refinement. The need for refinement is based on the risk contribution factor (RCF) of a zone:

$$
R C F_{i}=\frac{P\left[F_{i}\right]}{P_{d i s k}}
$$

Zones are refined by manually partitioning the domain into 2 or 4 sub-zones for surface and embedded zones, respectively.

The zone refinement process is a potentially timeconsuming process that is performed by a human. Therefore, an adaptive risk refinement methodology (ARRM) was developed to reduce human involvement in the zone discretization process. ARRM includes an adaptive nodal selection feature designed to identify the optimal distribution of nodes on the surface of the component, and it employs constrained Delaunay triangulation (CDT) to connect the nodes into an initial mesh. ARRM numerically integrates the surface of the initial mesh to estimate the cumulative conditional POF. The initial mesh is repeatedly refined until a converged disk risk solution is obtained.

\section{Background}

For illustrative purposes, the POF associated with an impeller rotor disk model (Fig. 2a) was obtained using a large number of zones. The POF for each zone represents the conditional POF before 20,000 fatigue cycles given an anomaly originating in that zone. The resulting conditional POF surface shown in Fig. 2b serves as a benchmark for assessing the effectiveness of ARRM.

Development of a zone discretization methodology focuses on the conditional POF in rotor disks. Conditional POF responses are high along edges associated with high stresses and abruptly diminish towards the interior of a disk. This generates steep gradients along rotor disk edges as illustrated in the benchmark impeller surface (Fig. 2b). From a modeling perspective, the slope of the gradient is the most important aspect of rotor disk conditional POF behavior because it is difficult to develop a response surface for steep gradients.

Several methods were considered for modeling the conditional POF response, including Ordinary Kriging (OKR), multivariate adaptive regression splines (MARS), artificial neural networks (ANN), and polynomial regression (PR). . $^{3-7}$ MARS and ANN are capable of modeling steep gradients associated with rotor disk conditional POF behavior. However, they require a substantially large number of training points making them

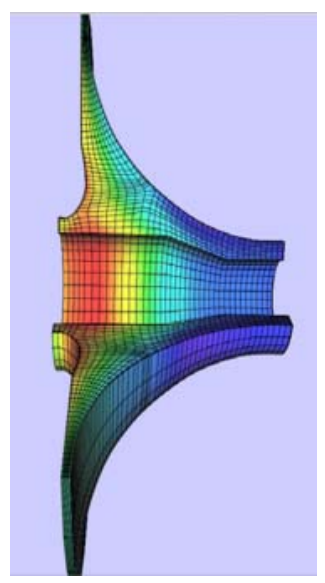

(a)

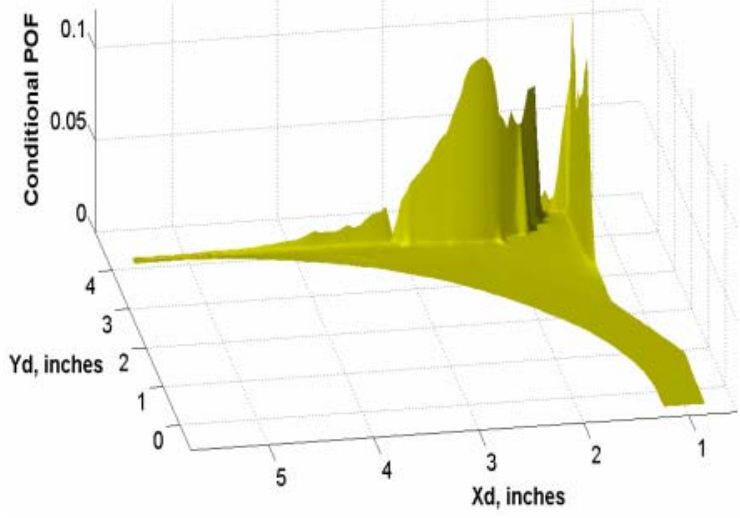

(b)
Figure 2. (a) Impeller rotor disk, and (b) Benchmark conditional POF surface based on 1150 nodal coordinates at the life limiting location of each zone. 


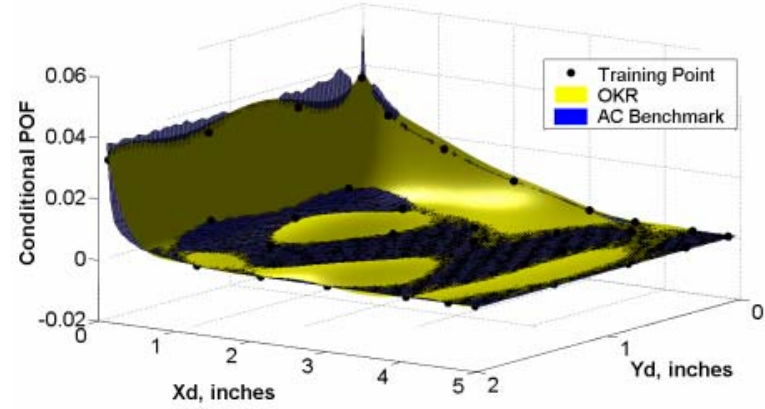

(a)

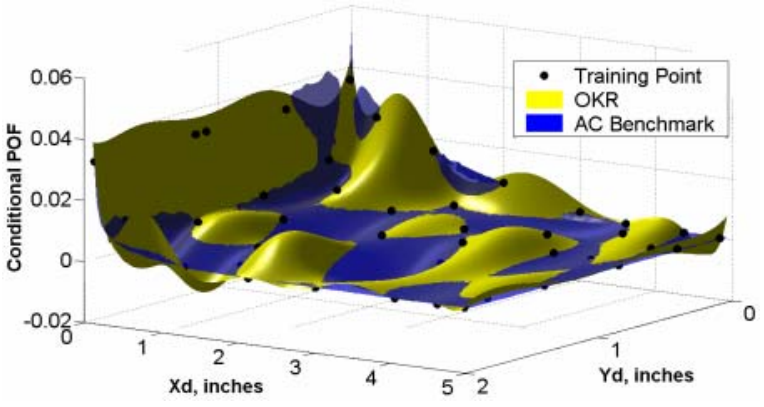

(b)

Figure 3. The accuracy of the OKR model may actually decrease with an increase in the number of training points. (a) 40 training points, and (b) 50 training points.

computationally expensive. A training point is a nodal response required by a metamodel to build a response surface from which the response at other coordinates can be estimated. PR fails to generate successful models because its data smoothing nature inhibits it from characterizing abrupt response changes. OKR can model steep gradients using relatively few training points. However, as shown in Fig. 3, the accuracy of the OKR model may actually decrease with an increase in the number of training points.

\section{Initial Mesh Generation}

The initial mesh is based on the nodal coordinates of a finite element model. Mesh generation consists of three steps: (1) surface node selection, (2) embedded node selection, and (3) constrained Delaunay triangulation (CDT). Surface nodes are selected with the intention to accurately model conditional POF along a rotor disk surface using a minimal number of nodes.

\section{Surface Node Selection}

The surface node selection process begins with the identification of an initial nodal set. This set is defined as the coordinates of surface nodes located at gradient reversals along the delta stress response curve shown in Fig. 4. The POF is predicted at each of the initial approach node locations using the critical defect size (CDS), where CDS is an efficient method for computing the conditional POF $^{1}$ (Fig. 5). Not every member in the nodal set is needed to characterize the CDS response curve. Some nodes can be removed without a significant change in the response as shown in Fig. 6. The area under the estimated CDS curve differs less than 1\% from the area under the estimated CDS curve in Fig. 5. Estimated CDS curves are created by connecting neighboring nodes using one-dimensional linear elements.

The estimated CDS curve is refined through a process called curve refinement, whereby substandard elements are identified and subdivided. A substandard element is an element whose accuracy falls below a threshold value, which is selected depending upon the desired level of accuracy for the refined curve. Accuracy is quantified using the relative difference between the area underneath an element and the area under the actual CDS response curve encompassed by the element's endpoints. The actual CDS response curve is unknown; therefore, an estimate of the actual curve's area is used. It is obtained by computing the CDS response at an element's bisection coordinate,

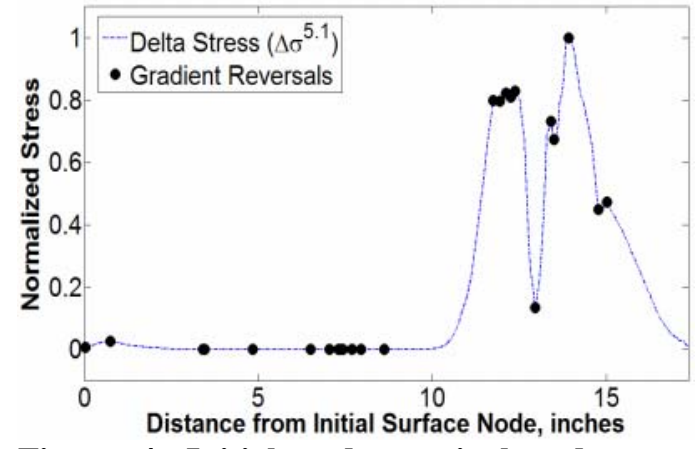

Figure 4. Initial node set is based on stress gradient reversals along the surface of the disk.

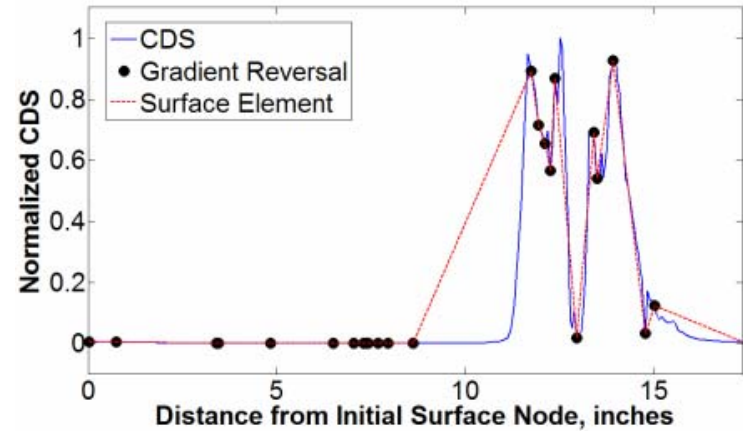

Figure 5. The POF is predicted at each node using the CDS approach. 


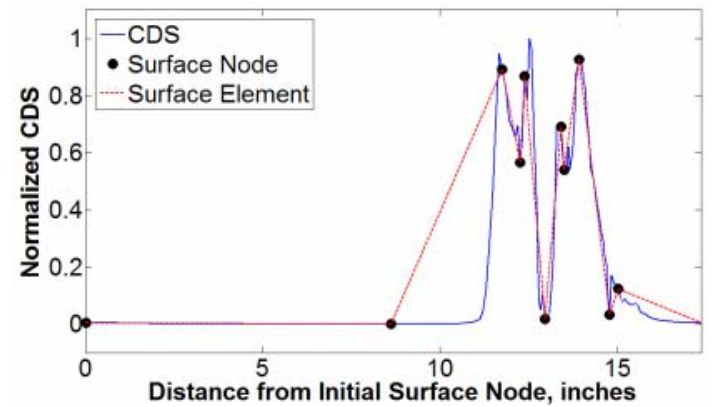

Figure 6. Nodal reduction of estimated CDS response curve.

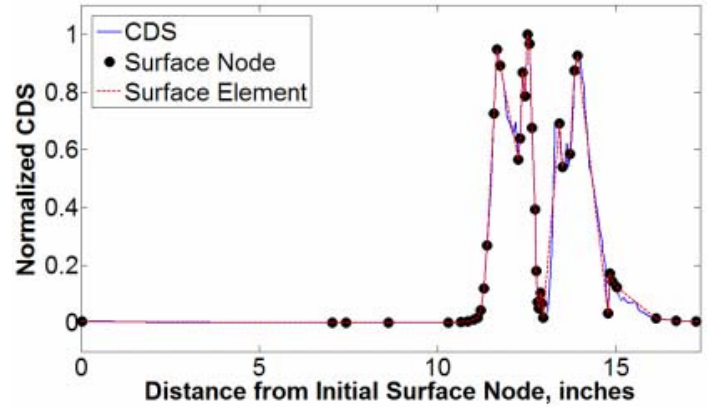

Figure 7. Curve refinement improves the accuracy of the estimated CDS response curve.

subdividing the element at the bisection node, and computing the cumulative area underneath the resulting subelements. Substandard elements are replaced by their sub-elements. Figure 7 illustrates the result of curve refinement applied to the estimated CDS response curve.

Again, the nodal set passes through the nodal reduction process (Fig. 8a). This concludes identification of the nodal set essential to the characterization of conditional POF along a rotor disk surface. However, as shown in Fig. $8 \mathrm{~b}$, the nodal set does not address the rotor disk geometric profile. Therefore, geometry-based nodes are appended to the response-based nodal set.

Geometry-based nodes are obtained through skeletonization and geometric refinement. Skeletonization is a method that provides the thinnest representation of a geometric outline (skeleton) that preserves the topology. Although many skeletonization techniques exist ${ }^{8-11}$, ARRM approximates rotor disk skeletons by identifying the Voronoi diagram generated using the finite element model surface nodes. The interior segments in the Voronoi diagram provide the rotor disk skeleton. Figure 9a is the Voronoi diagram of the impeller rotor disk, and Fig. 9b shows the corresponding skeleton, as well as its endpoints. The skeleton endpoints are important because they identify the apexes of geometric convexities within a rotor disk. Consequently, adding the nodal coordinates of the skeleton endpoints to the response-based nodal set creates an estimated surface model that encompasses the rotor disk. Figure 10a illustrates the estimated model consisting of both the response-based nodes and skeleton endpoints.

To improve geometric accuracy, the estimated surface model is refined with respect to the rotor disk geometry using curve refinement. Element accuracy is assessed using the relative error between the length of an element and the length of the actual rotor disk edge encompassed by the element's endpoints. Substandard elements are determined by the relative error threshold. A lower threshold results in a more accurate depiction of the rotor disk geometry. Substandard elements are subdivided at the finite element model surface node whose Euclidean distance to its relative location along the element is greatest. The process is repeated until all elements satisfy the threshold value. The result is shown in Figure 10b.

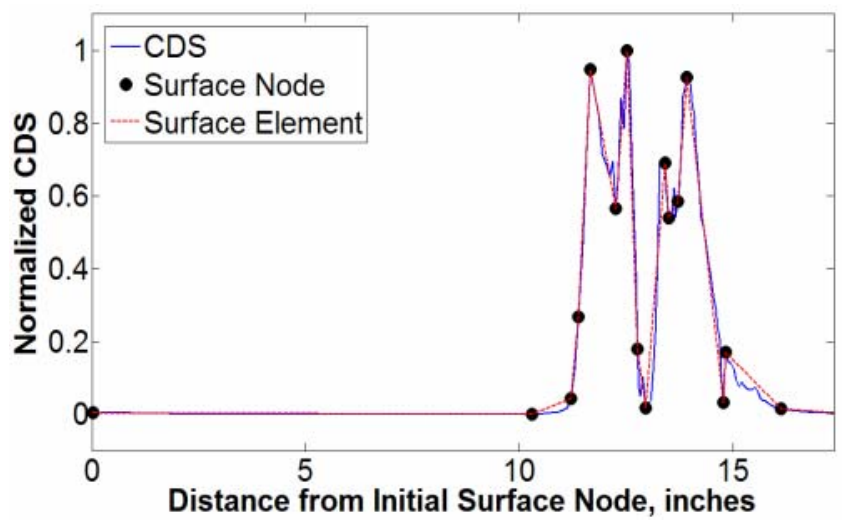

(a)

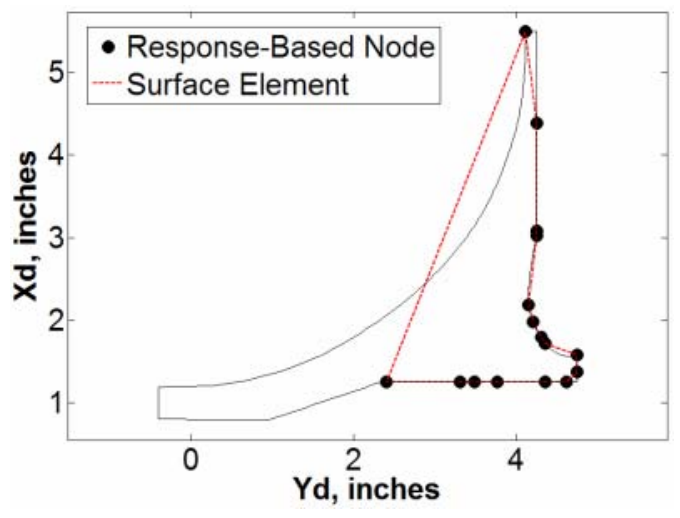

(b)

Figure 8. (a) Nodal reduction is applied a second time to the refined estimated CDS curve, (b) Responsebased nodes plotted in $2 \mathrm{D}$ geometric plane fail to characterize rotor disk geometry. 


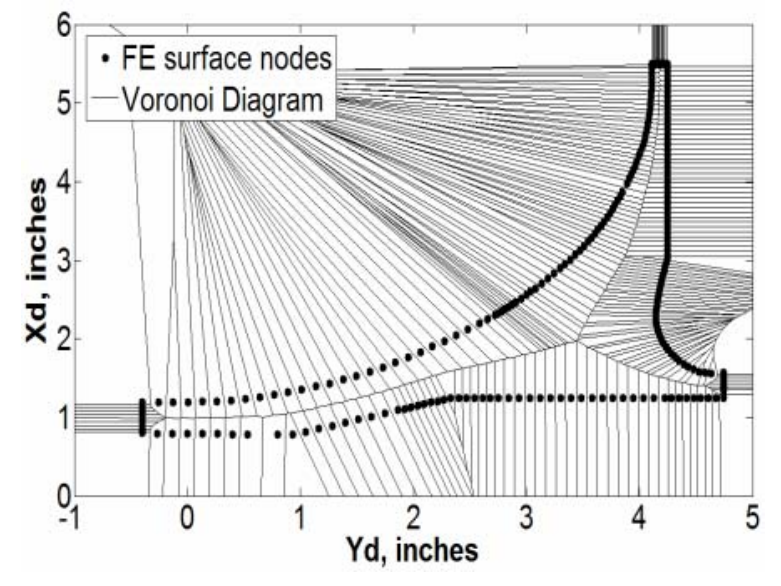

(a)

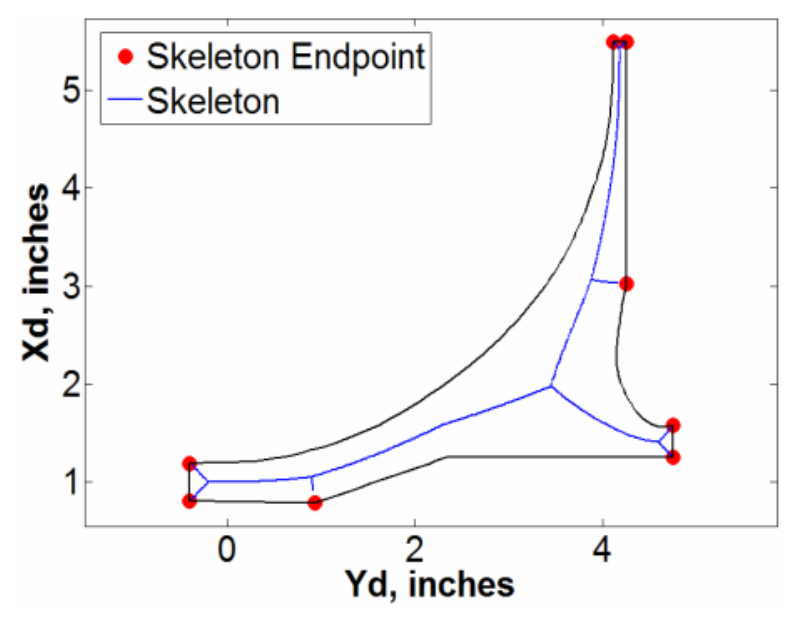

(b)

Figure 9. (a) Voronoi diagram based on the impeller finite element model surface nodes, (b) Skeleton and corresponding endpoints.

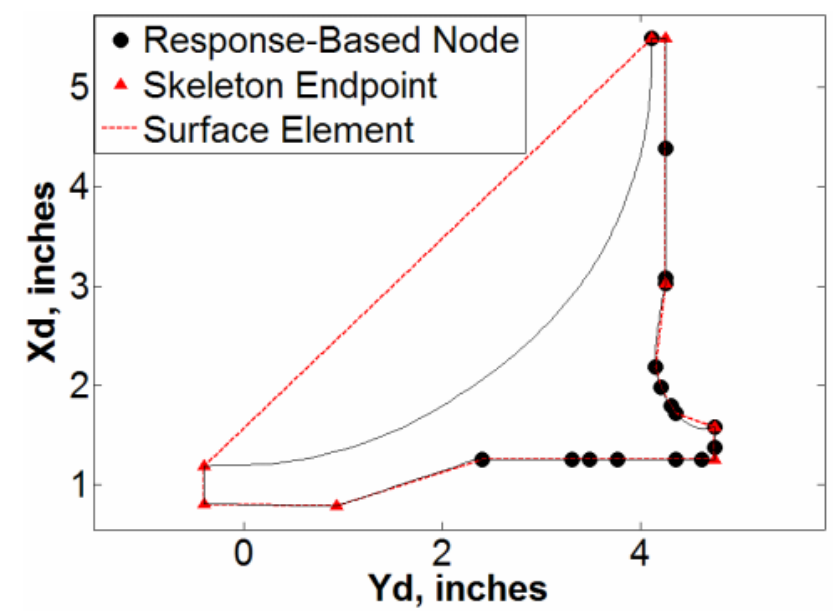

(a)

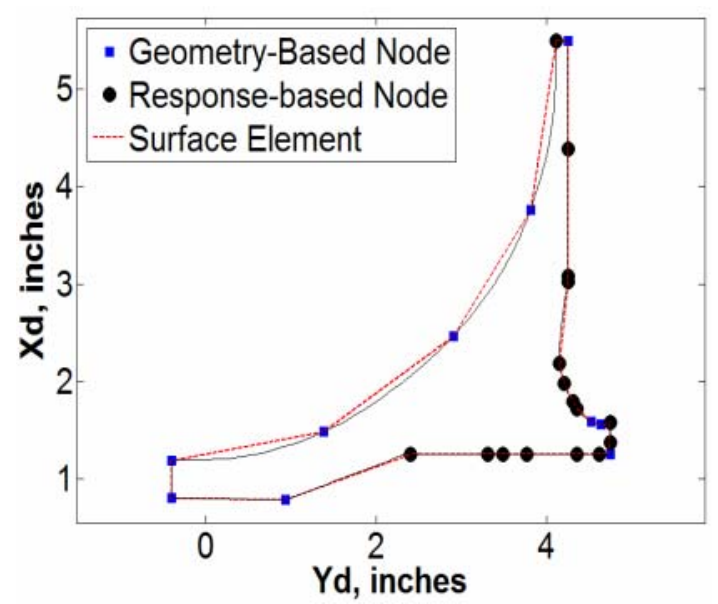

(b)

Figure 10. (a) Addition of skeleton endpoints to response-based nodes provides a model that encompasses the rotor disk, (b) Geometric refinement of estimated surface model completes characterization of rotor disk geometry.

\section{Embedded Node Selection}

In an effort to reduce conservatism in the initial mesh, ARRM adds embedded nodes to the nodal set composed of only surface nodes. Distributed judiciously, embedded nodes can restrain high conditional POF responses within small elements along a rotor disk edge. In turn, the accuracy of the initial mesh is improved. ARRM creates thin mesh surface elements where large conditional POF gradients are present. Definition of the embedded node coordinates requires projection paths along which nodes can exist. A projection path is the shortest route from a surface node to a lower bound determined through a mapping procedure. The mapping procedure calculates the equivalent coordinate along a skeleton for a given surface node. Using a skeleton as the basis for the lower bound prevents projection paths from intersecting one another as shown in Fig. 11. For computational efficiency, embedded nodes are only defined for surface nodes whose CDS response exceeds a threshold value, (i.e. 10\% of the maximum CDS response in the nodal set). 
An iterative search routine identifies the coordinates of embedded nodes along each projection path for selected surface nodes. The iterative search routine begins by computing the CDS at the lower bound. If the response is below the threshold value, the coordinate shifts along the projection path towards the rotor disk edge. Otherwise, the coordinate shifts towards the skeleton. This step continues until a specified number of attempts or the threshold value is reached. Each attempt reduces the previous shift distance by a factor of two:

$$
d_{s}=\left(\frac{l_{e}}{2}\right) \cdot 2 \exp [-m \cdot \ln (2)]
$$

where $d_{s}$ is the shifting distance, $l_{e}$ is the length of the projection path, and $m$ is the attempt number. Figure 12 shows the selected embedded nodes for the impeller using the iterative search routine.

\section{Constrained Delaunay Triangulation}

ARRM constructs an initial mesh using the adaptively selected nodes. Meshing is carried out using an advanced form of Delaunay triangulation known as CDT. ${ }^{12}$ Delaunay triangulation is a meshing technique whereby the likelihood of generating degenerate, or highly obtuse, triangular elements is minimized by maximizing the minimum angle of all mesh elements. ${ }^{13}$ However, along convex edges, Delaunay triangulation is unable to prevent the generation of elements that fall outside of the rotor disk geometry. CDT prevents the generation of exterior elements. Figure 13 illustrates the initial mesh generated for the impeller rotor disk using CDT.

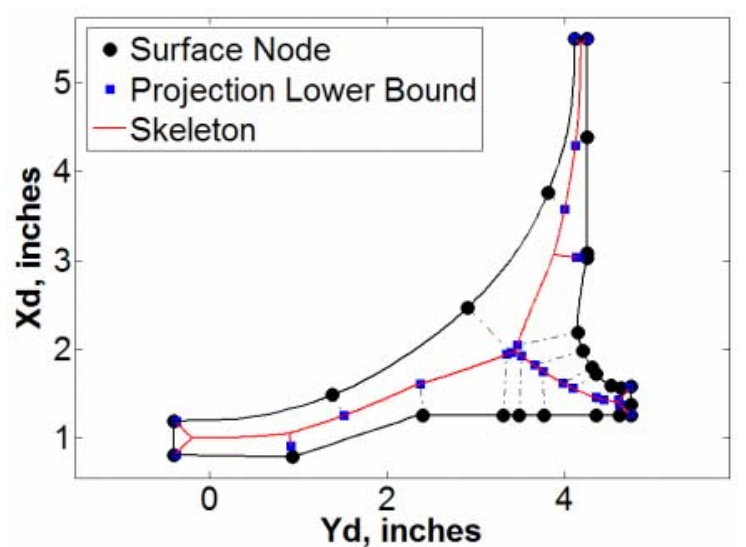

Figure 11. Projection paths begin at surface nodes and end at the skeleton.

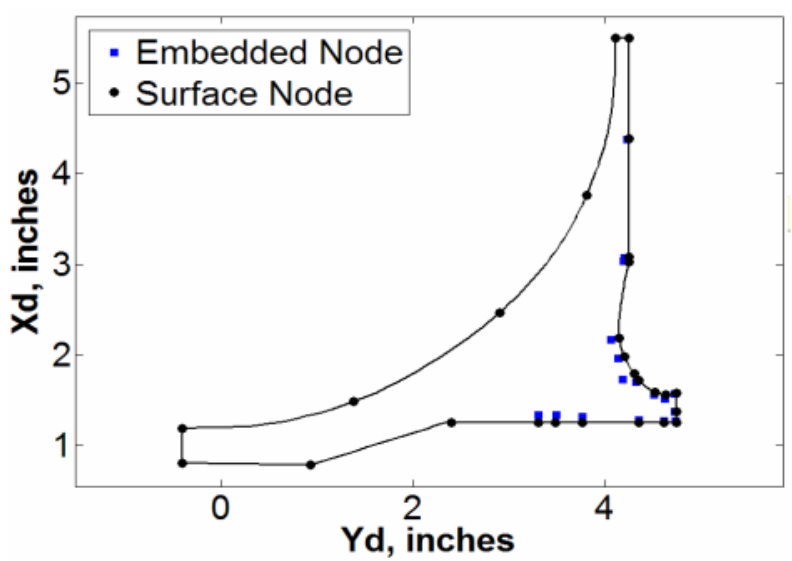

Figure 12. Iterative search routine provides nodal coordinates for embedded nodes.

\section{Cumulative POF Estimation}

The cumulative POF is estimated by numerically integrating the conditional POF surface of the rotor disk mesh. For the surface model of a mesh, ARRM uses superparametric elements. ${ }^{14-17}$ Superparametric elements refer to elements whose interpolation shape functions are of a lesser order than their geometric shape functions. ARRM employs linear interpolation shape functions and quadratic geometric shape functions. This results in a linear surface, which guarantees conservatism, and a more accurate geometric fit. The superparametric surface mesh of the impeller rotor disk is shown in Fig. 14a. It strongly resembles the benchmark impeller surface shown in Fig. 14b.

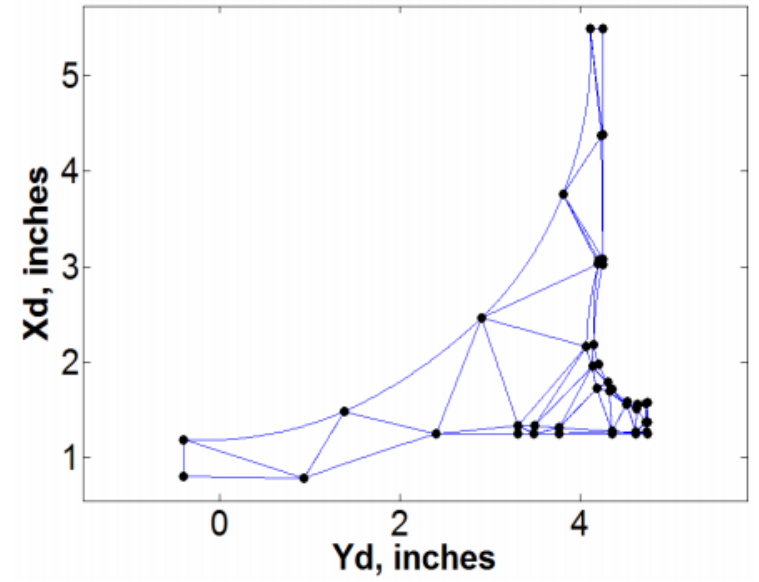

Figure 13. CDT using adaptively defined initial mesh nodal set. 


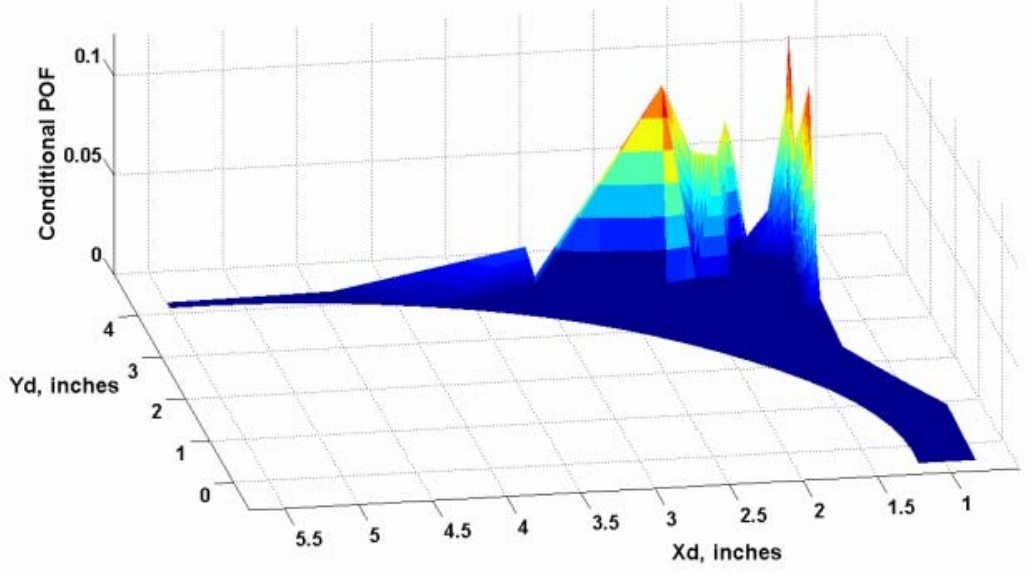

(a)

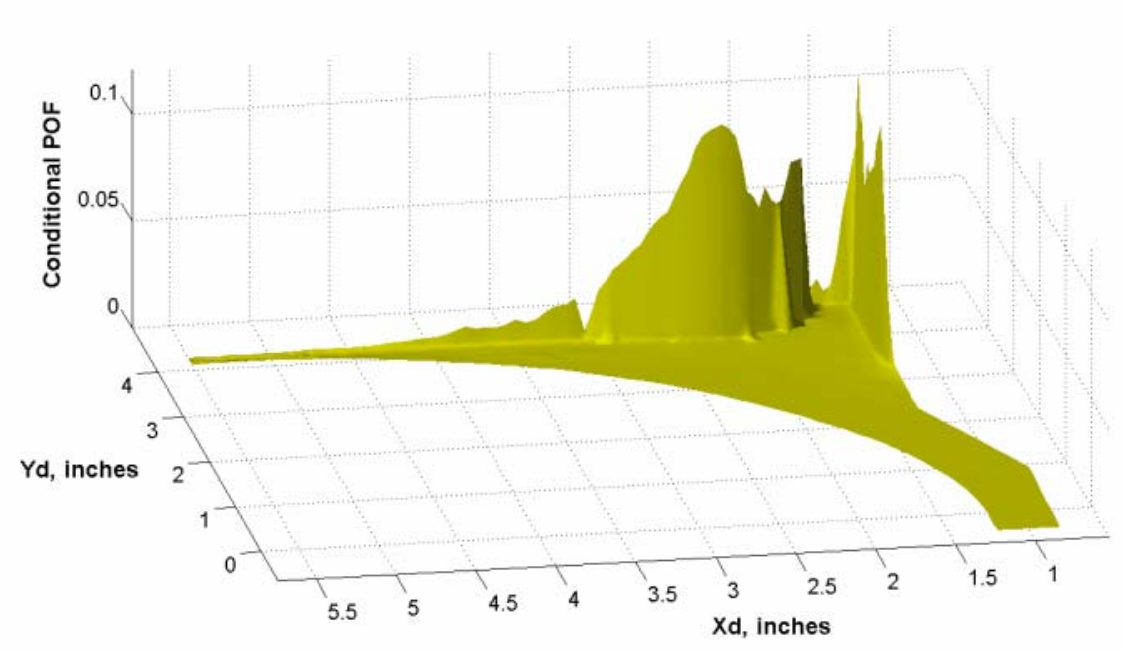

(b)

Figure 14. Conditional POF response surfaces for two underlying computational models: (a) Superparametric surface mesh, and (b) Zone-based mesh (benchmark).

\section{Adaptive Mesh Refinement}

To obtain a converged disk risk solution, the initial mesh is further refined using adaptive mesh refinement (AMR) ${ }^{18-20}$ AMR determines the appropriate distribution of the degree of refinement throughout a pre-defined mesh. Conditioned appropriately, AMR refines an initial mesh using a minimal number of iterations. This is accomplished through an iterative assessment and subdivision process, where refinement is based on RCF and longest-edge bisection is employed for subdivision. Longest-edge bisection partitions an element into two subelements by connecting the midpoint of the longest edge to the opposite vertex. ${ }^{18}$ In turn, the angles of the subsequently refined triangulations are greater than or equal to half of the smallest angle in the initial element. LEB guarantees the construction of non-degenerate and smooth unstructured triangulations. Initially, all elements are assessed. Elements that satisfy the RCF threshold remain intact, and elements that fail are subdivided. The process continues until all elements satisfy the RCF threshold. Figure 15 illustrates the sequence of refinements for the impeller mesh using a $1 \%$ RCF threshold. RCF focuses refinements along the rotor disk edge where high-risk elements are present. 


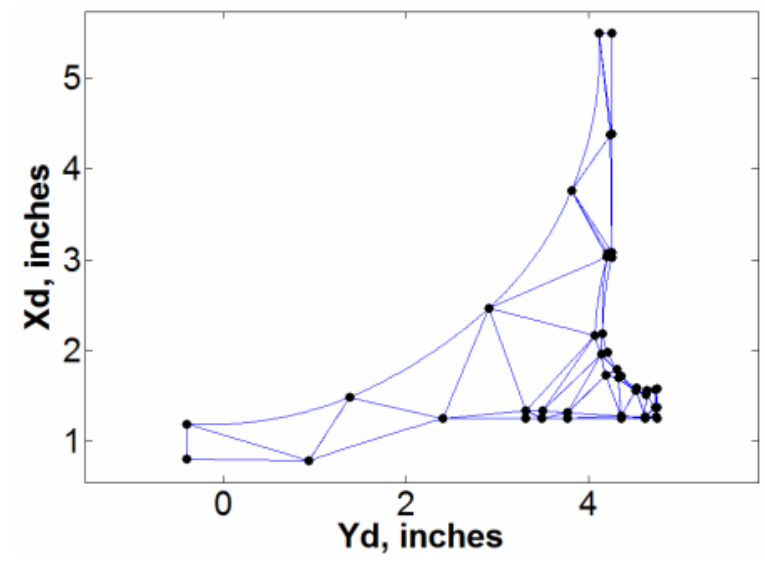

(a)

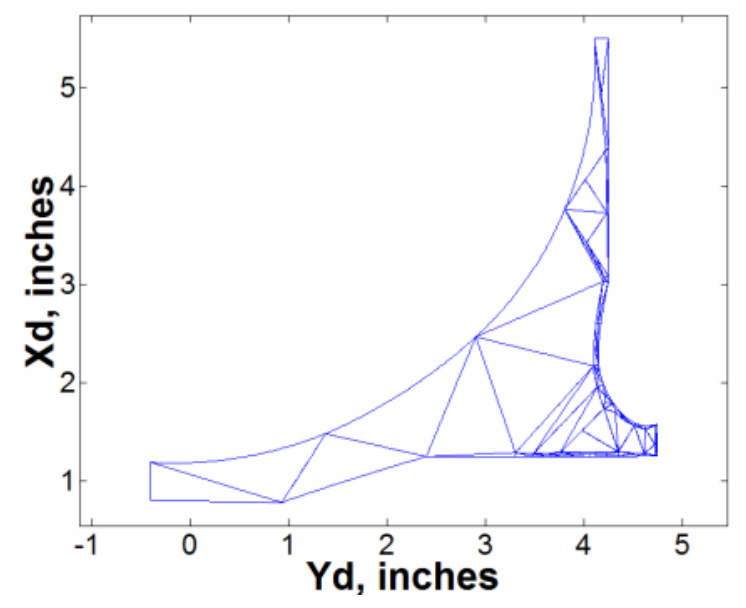

(c)

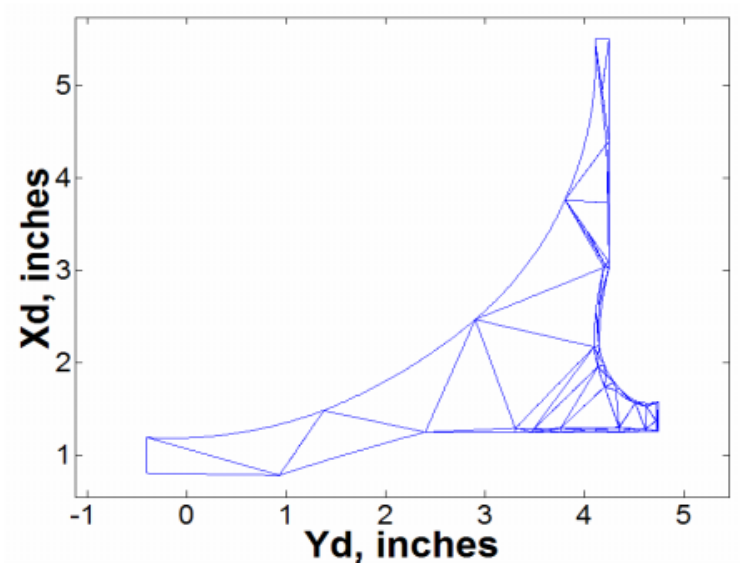

(b)

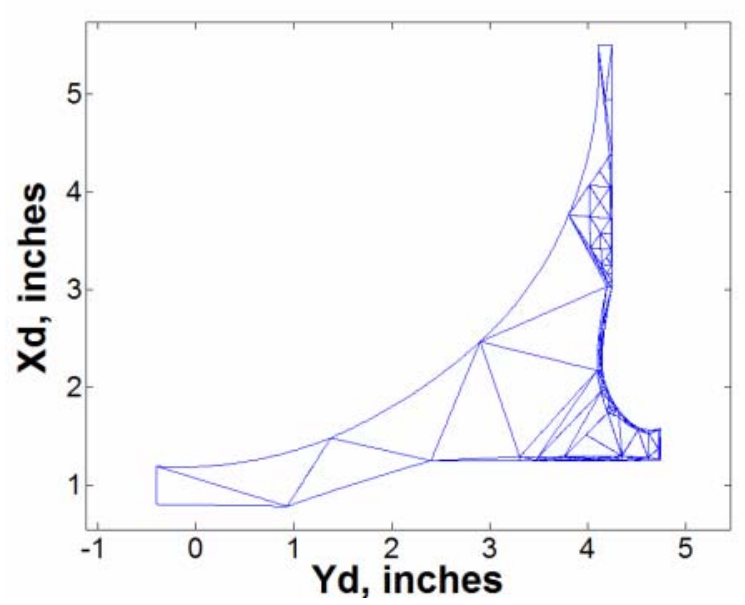

(d)

Figure 15. Adaptive mesh refinement sequence using 1\% RCF threshold. a) initial mesh: 43 nodes, b) first refinement: 54 nodes, c) second refinement: 69 nodes, d) eighth and final refinement: 119 nodes.

\section{Results}

Figure 16 shows the convergence of cumulative conditional POF with respect to the number of mesh nodes. ARRM yields a conservative converged solution which is approximately 1.8 times greater than the benchmark cumulative conditional POF solution. Conservatism in the final solution is arguably high. However, the solution obtained using ARRM is favorable in relation to the solution obtained from a 64-zone risk analysis model developed in a probabilistic fracture mechanics code.

In AMR, RCF directs refinement towards surface elements possessing high conditional POF responses. However, though the risk contribution is

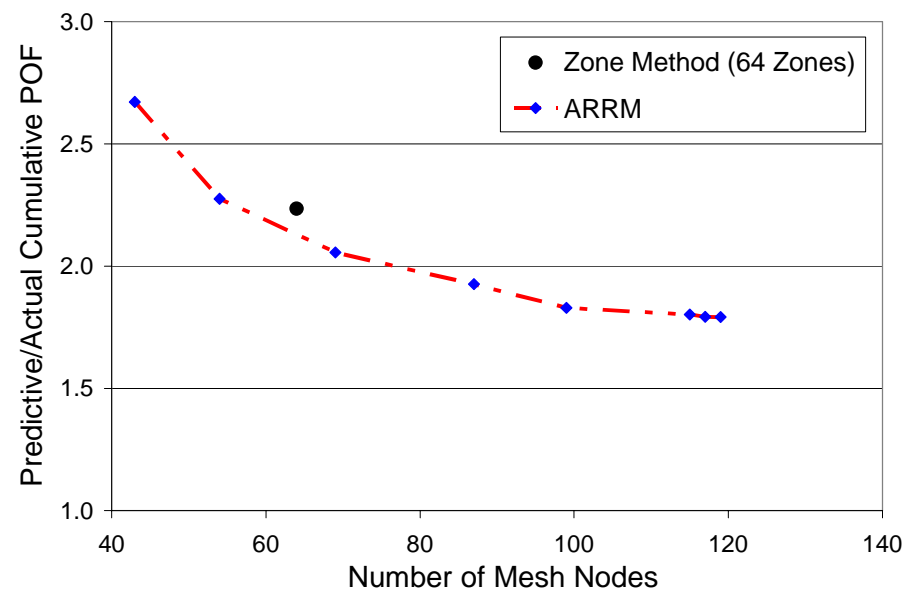

Figure 16. Convergence of cumulative conditional POF. 
high in these elements, the corresponding response surface is fairly accurate in relation to the benchmark model's surface. Subsequently, ARRM continually refines elements whose subdivision trivially improves the accuracy of the cumulative POF estimation. This is called over-refining and it is the predominant source of computational inefficiency in ARRM. It is also the primary source of conservatism. Because high-risk elements are targeted, gradient-containing elements with lower risk contributions are prone to being overlooked. Gradient-containing elements account for the majority of the conservatism in the superparametric surface. All things considered, an ideal alternative to RCF is an error-based assessment metric that quantifies the difference between the surfaces of the estimated superparametric surface and the actual surface.

Another source of conservatism originates from initial mesh faults. Recall that ARRM adds embedded nodes to the initial mesh in order to create thin surface elements that restrain high conditional POF within small localized regions. However, CDT does not guarantee the construction of the intended surface elements. In the instance that CDT does not create a surface element, the response corresponding to a high-risk surface node may extend across the rotor disk domain as shown in Fig. 17. Element $\boldsymbol{A B C}$ consists of a high-risk surface node $\boldsymbol{A}$, an embedded node $\boldsymbol{B}$, and a low-risk surface node $\mathbf{C}$. The conditional POF response at $\boldsymbol{A}$ propagates through the entire element resulting in an over-prediction of the cumulative POF. Ideally, CDT creates segment $\boldsymbol{B D}$. The result is an initial mesh with elements $\boldsymbol{A B D}$ and $\boldsymbol{D B C}$. The ideal mesh restrains the conditional POF associated with node $\boldsymbol{A}$ within the small surface element $\boldsymbol{A B D}$. The effect is a less conservative and more accurate estimate of the cumulative POF.

\section{Conclusion}

This paper focuses on modeling conditional POF behavior of gas turbine engine rotor disks. An adaptive risk refinement methodology was presented that consists of a sequence of processes that optimize the accuracy and efficiency of risk predictions. While the adaptive mesh refinement process provides converged risk solutions, it has some shortcomings. In particular, RCF over-refines high-risk surface elements and fails to sufficiently refine gradient-containing elements. Consequently, the converged risk solution is over-conservative. Future research will focus on replacing RCF with a more efficient assessment metric that directs refinement towards gradient-containing elements. Furthermore, a new constraint will be imposed in conjunction with CDT to prevent surface element mesh faults, a major source of conservatism in the initial mesh.

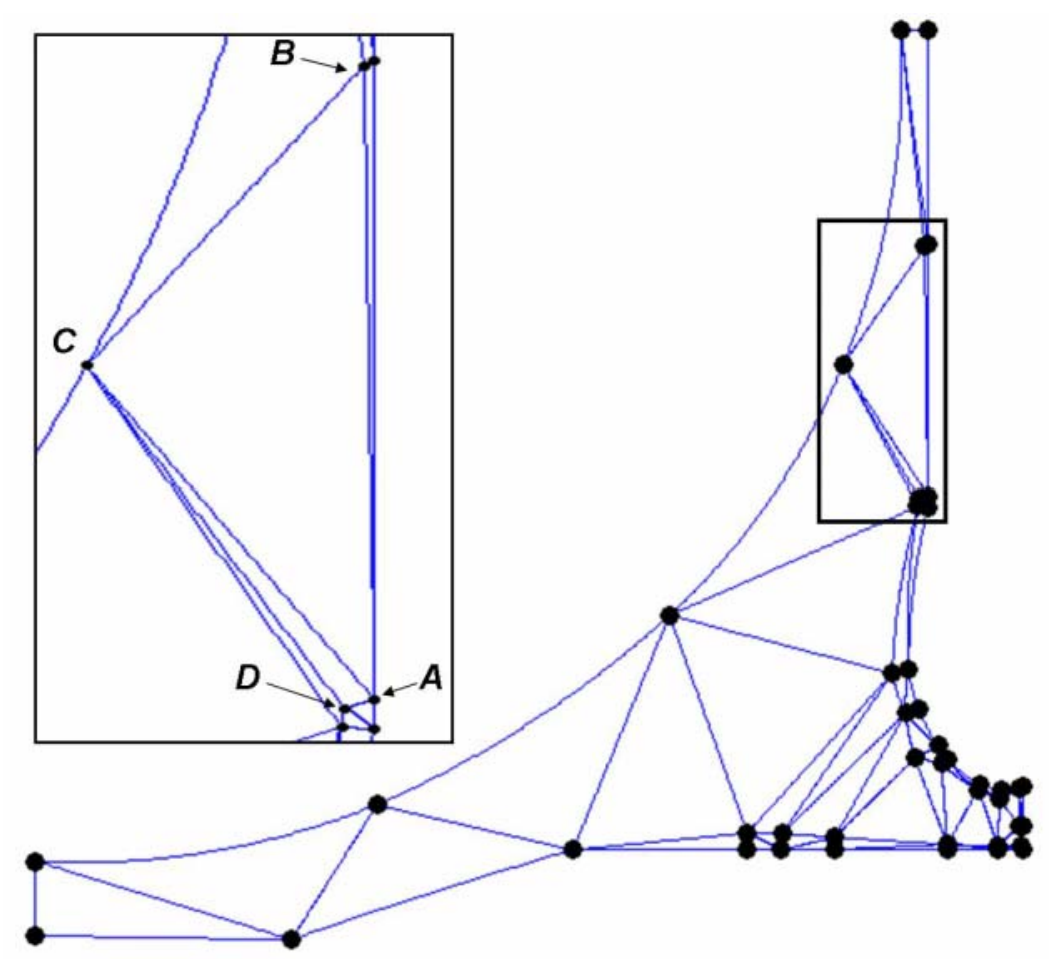

Figure 17. The response associated with high-risk surface nodes may extend into the rotor disk internal domain which may lead to over-prediction of disk risk. 


\section{Acknowledgements}

This work is performed as part of the Probabilistic Design for Rotor Integrity (PDRI) project supported by the Federal Aviation Administration under Grant 99-0-016. The authors wish to thank Joe Wilson (FAA William J. Hughes Technical Center project manager) and Tim Mouzakis (FAA Engine and Propeller Directorate, New England Regional Office) for their continued support. The collective contributions of the PDRI program team (Southwest Research Institute, GE Aviation, Honeywell, Pratt \& Whitney, Rolls-Royce Corporation, Mustard Seed Software, University of Texas at San Antonio) are gratefully acknowledged.

\section{References}

${ }^{1}$ Leverant, G. R., Millwater, H. R., McClung, R. C., and Enright, M. P., "A New Tool for Design and Certification of Aircraft Rotors," Journal of Engineering for Gas Turbine and Power, Vol. 126, No. 1, 2004, pp. 155-159.

${ }^{2}$ Millwater, H. R., Enright, M. P., and Fitch, S. H. K., "A Convergent Zone-Refinement Method for Risk Assessment of Gas Turbine Disks Subject to Low-Frequency Metallurgical Defects," Journal of Engineering for Gas Turbines and Power, Vol. 129, No. 3, 2007, pp. 827-835.

${ }^{3}$ Simpson, T., Peplinski, J., Koch, P., and Allen, J., "Metamodels for Computer-Based Engineering Design: Survey and Recommendations," Engineering with Computers, Vol. 17, No. 2, 2001, pp. 129-150.

${ }^{4}$ Myers, R. and Montgomery, D., Response Surface Methodology, John Wiley \& Sons, Inc., Canada, 2002.

${ }^{5}$ Yang, R. J., Wang, N., Tho, C., Bobineau, J. P., and Wang, B. P., "Metamodeling Development for Vehicle Frontal Impact Simulation," Transactions- American Society of Mechanical Engineers Journal of Mechanical Design, Vol. 127, 2005, pp. 10141020 .

${ }^{6}$ Cressie, N. A. C., Statistics for Spatial Data, John Wiley \& Sons, Inc., New York, 1993.

${ }^{7}$ Santner, T. J., Williams, B. J., and Notz, W. I., The Design and Analysis of Computer Experiments, Springer-Verlah, New York, 2003.

${ }^{8}$ Reddy, J. M. and Turkiyyah, G. M., "Computation of 3d Skeletons Using a Generalized Delaunay Triangulation Technique," Computer-Aided Design, Vol. 27, No. 9, 1995, pp. 677-694.

${ }^{9}$ Kimmel, R., Shaked, D., and Kiryati, N., "Skeletonization Via Distance Maps and Level Sets," Computer Vision and Image Understanding, Vol. 62, No. 3, 1995, pp. 382-391.

${ }^{10}$ Sudhalkar, A., Gursoz, L., and Prinz, F., "Box-Skeletons of Discrete Solids," Computer-Aided Design, Vol. 28, No. 6/7, 1996, pp. 507-517.

${ }^{11}$ Ivanov, D., Kuzmin, E., and Burtsev, S., "An Efficient Integer-Based Skeletonization Algorithm," Computers \& Graphics, Vol. 24, 2000, pp. 41-51.

${ }^{12}$ Shewchuk, J. R., "Delaunay Refinement Algorithms for Triangular Mesh Generation," Computational Geometry: Theory and Applications, Vol. 22, No. 1-3, 2002, pp. 21-74.

${ }^{13}$ Phongthanapanich, S. and Dechaumphai, P., "Adaptive Delaunay Triangulation with Object-Oriented Programming for Crack Propagation Analysis," Finite Elements in Analysis and Design, Vol. 40, No. 13-14, 2004, pp. 1753-1771.

${ }^{14}$ Raamachandran, J., Boundary and Finite Elements, CRC Press, Boca Raton, 2000.

${ }^{15}$ Barrett, K. E., "Multilinear Jacobians for Isoparametric Planar Elements," Finite Elements in Analysis and Design, Vol. 40, 2004, pp. 821-853.

${ }^{16}$ Dhatt, G. and Touzot, G., The Finite Element Method Displayed, J. Wiley \& Sons, New York, 1984.

${ }^{17}$ Lancaster, P. and Salkauskas, K., Curve and Surface Fitting: An Introduction, Academic Press Inc., London, 1986.

${ }^{18}$ Suárez, J. P., Plaza, Á., and Carey, G. F., "The Propagation Problem in Longest-Edge Refinement," Finite Elements in Analysis and Design, Vol. 42, 2005, pp. 130-151.

${ }^{19}$ Fernandez, F. A., Yang, Y. C., and Ettinger, R. D., "A Simple Adaptive Mesh Generator for 2-D Finite Element Calculations," IEEE Transactions on Magnetics, Vol. 29, No. 2, 1993, pp. 1882-1885.

${ }^{20}$ Pica, A., "Geometric Mesh Adaptivity," Engineering Computations, Vol. 13, No. 5, 1996, pp. 4-12. 